\title{
Omega-3 PUFA Alters the Expression Level but Not the Methylation Pattern of the WIF1 Gene Promoter in a Pancreatic Cancer Cell Line (MIA PaCa-2)
}

\section{Babak Rahmani ${ }^{1}$. Dariush Hamedi Asl ${ }^{1}$. Taghi Naserpour Farivar ${ }^{2}$. Mehdi Azad ${ }^{3} \cdot$ Mehdi Sahmani $^{4} \cdot$ Nematollah Gheibi $^{2}$ (D)}

Received: 2 June 2018 / Accepted: 14 November 2018 / Published online: 16 January 2019 (c) Springer Science+Business Media, LLC, part of Springer Nature 2019

\begin{abstract}
Pancreatic cancer is the fourth leading cause of death in both males and females, with a 5-year relative survival rate of $8 \%$. The Wnt signaling pathway has a significant role in the pathogenesis of many tumors, including those of pancreatic cancer. Hypermethylation of the Wnt inhibitory Factor-1 (WIF1) gene promoter have been detected in different types of cancer. In contrast, the anticancer effects of long-chain omega-3 PUFA (ALA) have been reported. Regarding its anticancer effects, in this study, we investigated the effects of various concentrations of omega-3 PUFA on expression level and promoter methylation of the WIF1 gene in MIA PaCa-2 cells in 24,48 , and $72 \mathrm{~h}$ after treatment. MIA PaCa-2 cells were treated with different concentrations of omega-3 PUFA $(25,50,100,250,500$, and $1000 \mu \mathrm{M})$. Cell viability assay was carried out followed by quantitative reverse transcriptase-polymerase chain reaction (qRT-PCR) and methylation-specific PCR (MSP). This investigation suggested that dietary consumption of omega-3 PUFAs $(250-1000 \mu \mathrm{M})$ has a significant effect on the proliferation and WIF1 gene expression of the MIA PaCa-2 cancer cell line but no effect on the promoter methylation of this gene. Changes in promoter methylation were not observed in any of the treatments.
\end{abstract}

Mehdi Sahmani

m.sahmani@gmail.com

$\triangle$ Nematollah Gheibi

ngheibi@qums.ac.ir; gheibi_n@yahoo.com

1 Department of Molecular Medicine, Faculty of Medical Sciences, Qazvin University of Medical Sciences, Qazvin, Iran

2 Cellular and Molecular Research Centre, Qazvin University of Medical Sciences, Qazvin, Iran

3 Department of Medical Laboratory Sciences, Faculty of Allied Medicine, Qazvin University of Medical Sciences, Qazvin, Iran

4 Department of Clinical Biochemistry and Genetic, Faculty of Medicine, Qazvin University of Medical Sciences, Qazvin, Iran 